\title{
MAXIMAL MONOTONE OPERATORS IN NONREFLEXIVE BANACH SPACES AND NONLINEAR INTEGRAL EQUATIONS OF HAMMERSTEIN TYPE ${ }^{1}$
}

\author{
BY HAIM BREZIS AND FELIX E. BROWDER
}

Communicated by Alberto Calderón, March 19, 1974

Let $Y$ be a Banach space, $Y^{*}$ its conjugate space, $X$ a weak*-dense closed subspace of $Y^{*}$ with the induced norm. We denote the pairing between $x$ in $X$ and $y$ in $Y$ by $(y, x)$. If $T$ is a mapping from $X$ into $2^{Y}$, $T$ is said to be monotone if for each pair of elements $[x, y]$ and $[u, w]$ of $G(T)$, the graph of $T$, we have $(y-w, x-u) \geqq 0$. $T$ is said to be maximal monotone from $X$ to $2^{Y}$ if $T$ is monotone and maximal among monotone mappings in the sense of inclusion of graphs.

The theory of maximal monotone mappings has been intensively developed in the case in which $Y$ is reflexive and $X=Y^{*}$. In this note, we present an extension of this theory to the case in which $X$ and $Y$ are not reflexive, and show that this extended theory can be used to give a new and more conceptual proof of a general existence theorem for solutions of nonlinear integral equations of Hammerstein type established by the writers in [2] by more concrete arguments.

An essential tool in our discussion is supplied by the following definitions:

Definition 1. Let $T$ be a mapping from $X$ into $2^{Y}$. Then $T$ is said to be $X$-coercive if for each real number $k$, the set $\{x \mid x \in X$, there exists $w$ in $T(x)$ such that $(w, x) \leqq k\|x\|\}$ is contained in a convex weak* compact subset $A_{k}$ of $X$.

THEOREM 1. Let $T$ be a monotone mapping from $X$ to $2^{Y}$. Suppose that 0 lies in $D(T)$, the effective domain of $T$, and that $T$ is $X$-coercive. Then the range $R(T)$ of $T$ is all of $Y$.

We use the following extension of the concept of pseudo-monotonicity [1]:

AMS (MOS) subject classifications (1970). Primary 47H05, 47G05; Secondary $47 \mathrm{H} 15$.

${ }^{1}$ Research supported by NSF grant GP-28148. 
Definition 2. Let $f$ be a mapping of $X$ into $Y$. Then $f$ is said to be pseudo-monotone from $X$ to $Y$ if the following three conditions are satisfied:

(a) $f$ is continuous from each finite-dimensional subspace of $X$ to the weak topology on $Y$ induced by the functionals from $X$.

(b) $f$ maps each weak* compact subset of $X$ into a subset of $Y$ which is compact with respect to the weak topology induced by $X$ on $Y$.

(c) Let $\left\{u_{y}\right\}$ be a filter base on $X$ contained in a weak* compact subset of $X$ and converging in the weak ${ }^{*}$-topology to an element $u$ of $X$, and suppose that $\lim \sup \left(f\left(u_{\gamma}\right), u_{\gamma}-u\right) \leqq 0$. Then $f\left(u_{\gamma}\right)$ converges to $f(u)$ in the weak topology on $Y$ induced by $X$ and $\lim \left(f\left(u_{\gamma}\right) u_{\gamma}\right)=(f(u), u)$.

THEOREM 2. Let $T$ be a maximal monotone mapping from $X$ to $2^{Y}$ which is $X$-coercive. Let fbe a pseudo-monotone mapping from $X$ to $Y$ in the sense of Definition 2, such that for some constant $c,(f(u), u) \geqq-c\|u\|$. Suppose that $0 \in D(T)$. Then the range of $(T+f)$ is all of $Y$.

LEMMA 1. Let $f$ be a monotone mapping from $X$ to $Y$ which satisfies conditions (a), (b) of Definition 2. Then $f$ is pseudo-monotone from $X$ to. $Y$.

COROLlaRY TO THEOREM 2. Let $T$ be a maximal monotone mapping from $X$ to $2^{Y}$ such that $T$ is $X$-coercive, $0 \in D(T)$. Let $f$ be a monotone mapping from $X$ to $Y$ satisfying conditions (a) and (b) of Definition 2. Suppose that for some constant $c$ and all $x$ in $X,(f(x), x) \geqq-c\|x\|$. Then the range of the mapping $(T+f)$ is all of $Y$.

In the case of reflexive Banach spaces, the proof of the corresponding existence theorems rests upon the following monotone extension theorem of Debrunner and Flor [4] (cf. [3] for the corresponding multivalued generalization and a conceptual development of the proof).

Proposition 1. Let $F$ be a finite subset of $X \times Y$ with $F$ monotone, i.e., $\left[u_{j}, w_{j}\right],\left[u_{k}, w_{k}\right]$ in $F$ implies that $\left(u_{j}-u_{k}, w_{j}-w_{k}\right) \geqq 0$. Let $C$ be the convex closure of the first components $\left\{u_{1}, \cdots, u_{n}\right\}$ of $F$, and suppose that $f$ is a continuous mapping from $C$ to $Y$ endowed with the weak topology induced by $X$. Then there exists an element $x$ of $C$ such that

$$
\left(w_{j}-f(x), u_{j}-x\right) \geqq 0, \quad(j=1, \cdots, n) .
$$

To prove our new existence theorems in the nonreflexive case, we use a modification of the Debrunner-Flor result given in Proposition 2 below, which is suggested by the results of Minty [5].

Proposition 2. Let $F$ be a finite monotone subset of $X \times Y$, and let $C_{0}$ be the convex closure of $\left\{0, u_{1}, u_{2}, \cdots, u_{n}\right\}$. Suppose that $f$ is a continuous mapping from $C_{0}$ to $Y$ endowed with the weak topology induced by $X$. Then there exists a point $x$ of $C_{0}$ of the form $x=\sum_{j=1}^{n} \xi_{j} u_{j}$, with $0 \leqq \xi_{j}, \sum_{j=1}^{n} \xi_{j} \leqq 1$, 
such that

and

$$
\left(w_{j}+f(x), u_{j}-x\right) \geqq 0, \quad(1 \leqq j \leqq n),
$$

$$
\sum_{j=1}^{n} \xi_{j}\left(w_{j}, u_{j}\right)+(f(x), x) \leqq 0 .
$$

Proof of Proposition 2. For the case in which $f \equiv 0$, the result was obtained by Minty in [5] by a direct argument. We obtain the more general result for nonzero $f$ by showing that Proposition 2 can be derived directly from Proposition 1. We note first that since the result really depends only upon the finite-dimensional space spanned by $\left\{u_{1}, \cdots, u_{n}\right\}$ and linear functionals upon this space, we may assume that $X=Y=H_{0}$, a finitedimensional Hilbert space. We may assume that $H_{0}$ is contained in a larger Hilbert space $H$, and that $H_{1}$ is an $n$-dimensional Hilbert space in $H$ which is orthogonal to $H$ and with orthonormal basis $\left\{h_{1}, \cdots, h_{n}\right\}$. Let $v_{j}=$ $u_{j}+h_{j}$ for each $j$, and let $C_{1}$ be the convex closure of $\left\{0, v_{1}, \cdots, v_{n}\right\}$. We form a new monotone set $F_{1}$ in $H \times H$ whose elements consist of $\left[v_{j}, w_{j}\right], 1 \leqq j \leqq n$, together with the single additional element $\left[0, w_{0}\right]$, where $w_{0}=\sum_{j=1}^{n}\left(w_{j}, u_{j}\right) h_{j}$. Let $\zeta$ be the mapping of $C_{1}$ into $C_{0}$ given by $\zeta\left(\sum_{j} \xi_{j} v_{j}\right)=\sum_{j} \xi_{j} u_{j}$, and let $g$ be the mapping of $C_{1}$ into $H$ given by $g(x)=$ $-f(\zeta(x))$.

We apply Proposition 1 to the monotone set $F_{1}$ and the mapping $g$. Then there exists an element $y$ in $C_{1}$ of the form $y=\sum_{j=1}^{n} \xi_{j} v_{j}$ with $0 \leqq \xi_{j}$, $\sum_{j=1}^{n} \xi_{j} \leqq 1$, such that for $1 \leqq j \leqq n$,

$$
\left(w_{j}-g(y), v_{j}-y\right) \geqq 0, \quad\left(w_{0}-g(y), 0-y\right) \geqq 0 .
$$

Since for each $j, w_{j}-g(y)$ lies in $H_{0}$, the first $n$ inequalities imply that if $x=\zeta(y)=\sum_{j=1}^{n} \xi_{j} u_{j}$, then $\left(w_{j}+f(x), v_{j}-x\right) \geqq 0$. For the last inequality, we see that $(g(y), y)=-(f(x), x)$, while

$$
\left(w_{0}, y\right)=\sum_{j=1}^{n}\left(w_{j}, u_{j}\right) \xi_{j}\left(h_{j}, h_{j}\right)=\sum_{j=1}^{n} \xi_{j}\left(w_{j}, u_{j}\right) .
$$

Hence, this inequality becomes

$$
\sum_{j=1}^{n} \xi_{j}\left(w_{j}, u_{j}\right)+(f(x), x) \leqq 0 . \quad \text { Q.E.D. }
$$

Theorem 1 is a special case of Theorem 2.

LEMMA 2. Let $X$ be a closed subspace of $Y^{*}, A$ a subset of $X$. Suppose that for each $\varepsilon>0$, there exists a weak* compact subset $A_{\varepsilon}$ of $X$ such that $A$

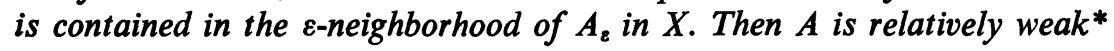
compact in $X$. 
Proof. Since each $A_{\varepsilon}$ must be bounded, $A$ is bounded in $X$ and hence relatively weak* compact in $Y^{*}$. Hence it suffices to show that the weak* closure $A_{1}$ of $A$ in $Y^{*}$ is contained in $X$. Since $A$ is contained in $A_{\varepsilon}+B_{\varepsilon}$, where $B_{\varepsilon}$ is the closed unit ball in $Y^{*}$, and since both $A_{\varepsilon}$ and $B_{\varepsilon}$ are weak* compact, it follows that $A_{1}$ must be contained in the weak* compact set $A_{\varepsilon}+B_{\varepsilon}$. Hence $A_{1}$ is contained in the $\varepsilon$-neighborhood of $X$ for each $\varepsilon>0$, i.e. $A_{1} \subset \bigcap_{\varepsilon>0} N_{\varepsilon}(X)=X$ since $X$ is a closed subspace of $Y^{*}$. Q.E.D.

Proof of Theorem 2. Since $0 \in D(T)$, we may add a constant to the values of $T$ and assume that $[0,0]$ lies in the graph of $T$ by absorbing the constant into the mapping $f$ without perturbing the pseudo-monotonicity of $f$. Similarly, if we wish to prove that an element $w_{0}$ of $Y$ lies in the range of $(T+f)$, it suffices to prove that 0 lies in $R\left(T+f_{0}\right)$, where $f_{0}(x)=f(x)-w_{0}$. Thus, it suffices to show that $0 \in R(T+f)$.

Let $F$ be a finite subset of $G(T)$, the graph of $T$. We apply Proposition 2 to $F=\left\{\left[u_{1}, w_{1}\right], \cdots,\left[u_{n}, w_{n}\right]\right\}$ to obtain a point $x_{F}=\sum_{j=1}^{n} \xi_{j} u_{j}$ with $\xi_{j} \geqq 0$ for each $j$, and $\sum_{j=1}^{n} \xi_{j} \leqq 1$ satisfying the two systems of inequalities:

$$
\begin{gathered}
\left(w_{j}+f\left(x_{F}\right), u_{j}-x_{F}\right) \geqq 0 \quad(j=1, \cdots, n), \\
\sum_{j=1}^{n} \xi_{j}\left(w_{j}, u_{j}\right)+\left(f\left(x_{F}\right), x_{F}\right) \leqq 0 .
\end{gathered}
$$

Corresponding to any $k>0$, we may group the set of first components of the subset $F$ into two classes, so that for $1 \leqq j \leqq r, u_{j}$ lies in the set $\{x \mid x \in X$, there exists $w$ in $T(x)$ such that $(w, x) \leqq k\|x\|\}$, and for $j>r, u_{j}$ lies outside this set. We set

$$
u_{k, F}=\sum_{j=1}^{r} \xi_{j} u_{j}, \quad v_{k, F}=\sum_{j=r+1}^{n} \xi_{j} u_{j}
$$

Since the elements of the first set of $u_{j}$ all lie by hypothesis in the weak* compact convex subset $A_{k}$ of $X$ and $0 \in A_{k}$, it follows that $u_{k, F}$ lies in $A_{k}$. Since $T$ is monotone, and by construction $0 \in T(0)$, it follows that for all $j,\left(w_{j}, u_{j}\right) \geqq 0$. Moreover, for all $x,(f(x), x) \geqq-c\|x\|$ by hypothesis. For $r+1 \leqq j \leqq n,\left(w_{j}, u_{j}\right) \geqq k\left\|u_{j}\right\|$. Hence

$$
k\left\|v_{k, F}\right\| \leqq k \sum_{j=r+1}^{n} \xi_{j}\left\|u_{j}\right\| \leqq \sum_{j=r+1}^{n} \xi_{j}\left(w_{j}, u_{j}\right) \leqq c\left\|x_{F}\right\| .
$$

If we apply the last inequality for a given value $k_{0}$ of $k, k_{0}>c$, we find that

$$
\left(k_{0}-c\right)\left\|v_{k_{0}, F}\right\| \leqq c\left\|u_{k_{0}, F}\right\|,
$$

while $u_{k_{0}, F}$ lies in the bounded subset $A_{k_{0}}$ of $X$. Hence, $\left\|v_{k_{0}, F}\right\| \leqq c_{0}$ independently of $F$, so that $\left\|x_{F}\right\| \leqq c_{1}$ independently of $F$. If we employ the 
same inequality for a general large $k$, we see that $\left\|v_{k, F}\right\| \leqq c c_{1} k^{-1}$ so that

$$
\operatorname{dist}\left(x_{F}, A_{k}\right) \leqq\left\|v_{k, F}\right\| \leqq c c_{1} k^{-1} \rightarrow 0 \quad(k \rightarrow \infty) .
$$

It follows from Lemma 2 that the set $A$ consisting of all solutions $x_{F}$ of the inequalities (1) and (2) above for finite subsets $F$ of $G(T)$ is contained in a weak* compact subset of $X$. By property (b) of Definition 2 for pseudomonotonicity of $f$, it follows that the set $B$ consisting of all elements of the form $f\left(x_{F}\right)$ is contained in a subset of $Y$ which is compact in the $X$-weak topology of $Y$. Hence if we consider the filter base consisting of $\left\{\left[x_{F}, f\left(x_{F}\right)\right]\right.$; $F$ containing $F_{1}$ \} for finite subsets $F_{1}$ of $G(T)$, the corresponding filter has an adherent point $\left[x_{0}, y_{0}\right]$ in $X \times Y$ in the product of the weak* topology on $X$ and the $X$-weak topology on $Y$. For any element $[x, y]$ in $F_{1}$ and $\left[x_{F}, f\left(x_{F}\right)\right]$ in the corresponding element of the filter base, we have $\left(y+f\left(x_{F}\right), x-x_{F}\right) \geqq 0$, i.e.,

$$
\left(f\left(x_{F}\right) x_{F}\right) \leqq\left(f\left(x_{F}\right), x\right)+\left(y, x-x_{F}\right) .
$$

Hence, taking limits on the filter,

$$
\begin{aligned}
\lim \sup \left(f\left(x_{F}\right), x_{F}-x_{0}\right) & \leqq \lim \left(f\left(x_{F}\right), x-x_{0}\right)+\left(y, x-x_{F}\right) \\
& \leqq\left(y_{0}, x-x_{0}\right)+\left(y, x-x_{0}\right)=\left(y_{0}+y_{3} x-x_{0}\right) .
\end{aligned}
$$

If the infimum of the bound on the right side for all elements $[x, y]$ of $G(T)$ is nonpositive, it follows from condition (c) of Definition 2 for the pseudomonotonicity of $f$ that $f\left(x_{0}\right)=y_{0}$ and $\lim \sup \left(f\left(x_{F}\right), x_{F}-x_{0}\right)=0$. It then follows that $\left(y_{0}+y, x-x_{0}\right) \geqq 0$ for all $[x, y]$ in $G(T)$, and since $T$ is maximal monotone, $-y_{0} \in T\left(x_{0}\right)$, i.e. $0 \in(T+f)\left(x_{0}\right)$. On the other hand, if $\left(y+y_{0}, x-x_{0}\right) \geqq \beta>0$ for all $[x, y]$ in $G(T)$, it follows that $-y_{0} \in T\left(x_{0}\right)$, and hence $\left(-y_{0}+y_{0}, x_{0}-x_{0}\right)=0 \geqq \beta>0$, which is a contradiction. Thus we have shown that 0 lies in $(T+f)(X)$, from which it follows as we have noted earlier that $Y=(T+f)(X)$. Q.E.D.

Theorem 2 may be applied to abstract Hammerstein equations through the following:

THEOREM 3. Let $f_{0}$ be a monotone mapping of $Y$ into $X$ which is continuous from finite-dimensional subspaces of $Y$ to the weak* topology of $X$, f a pseudo-monotone mapping of $X$ into $Y$, with $(f(x), x) \geqq-c\|x\|$ for a suitable constant $c$ and all $x$ in $X$. Suppose that $f_{0}$ maps bounded subsets of $Y$ into bounded subsets of $X, f_{0}\left(y_{0}\right)=0$ for some $y_{0}$ in $Y$, and $f_{0}$ satisfies conditions:

(1) $f_{0}$ is tricyclically monotone, i.e. for any three points $y, u$, and $v$ of $Y$, we have

$$
\left(f_{0}(y), y-u\right)+\left(f_{0}(u), u-v\right)+\left(f_{0}(v), v-y\right) \geqq 0 .
$$

(2) For any constant $k$, the set $\left\{f_{0}(y) ;\left(f_{0}(y), y\right) \leqq k,\left\|f_{0}(y)\right\| \leqq k\right\}$ is contained in a weak* compact convex subset of $X$. 
Then the range of the mapping $\left(I+f f_{0}\right)$ of $Y$ into $Y$ is the whole of the Banach space $Y$. In addition, if $f$ is monotone, then $\left(I+f f_{0}\right)$ is one-to-one and has a continuous inverse.

Proof of Theorem 3. For reasons of brevity, we shall only give the reduction of the existence assertion of Theorem 3 to the results of Theorem 2. Let $y_{1}$ be a given element of $Y$. If we seek to solve the equation $y+$ $f\left(f_{0}(y)\right)=y_{1}$, we introduce $u=f_{0}(y)$ as a new variable, and $y_{1} \in\left(f_{0}^{-1}+f\right)(u)$, and it follows easily that if this latter equation has a solution $u$, then so does the original equation. Since $f$ is pseudo-monotone from $X$ to $Y$ and satisfies the inequality $(f(u), u) \geqq-c\|u\|$, while $f_{0}^{-1}$ is the inverse of a maximal monotone mapping from $Y$ to $X$, and hence is itself maximal monotone from $X$ to $2^{Y}$, and $0 \in D\left(f_{0}^{-1}\right)$, the applicability of Theorem 2 is reduced to showing that the mapping $f_{0}^{-1}$ satisfies the basic hypothesis of $X$-coercivity.

We consider three points $y, u$, and $y_{0}$ in $Y$ with $f_{0}\left(y_{0}\right)=0$, and obtain the inequality

Hence

$$
\left(f_{0}(y), y-u\right)+\left(f_{0}(u), u-y_{0}\right) \geqq 0 \text {. }
$$

$$
\left(f_{0}(y), u\right) \leqq\left(f_{0}(y), y\right)+\left(f_{0}(u), u-y_{0}\right) .
$$

Taking the supremum of $\left(f_{0}(y), u\right)$ over all $u$ in the ball of radius $R$ about 0 in $Y$ and noting that $f_{0}$ is bounded on bounded sets, we see that

$$
R\left\|f_{0}(y)\right\| \leqq\left(f_{0}(y), y\right)+c(R) \quad(R>0) .
$$

Thus if $[u, y]$ lies in $G\left(f_{0}^{-1}\right)$, i.e., $u=f_{0}(y)$,

$$
R\|u\| \leqq(u, y)+c(R) .
$$

Hence if $(u, y) \leqq k\|u\|$, it follows that

$$
R\|u\|-c(R) \leqq k\|u\| \quad(R>k),
$$

from which it follows that $\|u\| \leqq s(k)$. Moreover, $u$ lies in the set $\left\{f_{0}(y)\right.$; $\left.\left\|f_{0}(y)\right\| \leqq s(k),\left(f_{0}(y), y\right) \leqq k s(k)\right\}$, which by condition (2) is contained in a weak* compact subset of $X$. Hence $f_{0}^{-1}$ is $X$-coercive and the existence result follows from Theorem 2. Q.E.D.

To use Theorem 3 to obtain an existence theorem for the Hammerstein integral equation

$$
u(t)+\int_{\Omega} k(t, s) h(s, u(s)) d s=v(t) \quad(t \in \Omega)
$$

as in [2], we choose $X$ to be $L^{1}(\Omega), Y=L^{\infty}(\Omega)$. The mapping $f$ is the monotone linear operator $(k u)(t)=\int_{\Omega} k(t, s) u(s) d s$, while $f_{0}$ is the Niemitskyii operator $\left(f_{0}(v)\right)(t)=h(t, v(t))$. The condition (1) that $f_{0}$ is tricyclically 
monotone follows from the fact that $f_{0}$ is a potential operator, while condition (2) follows immediately from the criterion of Dunford and Pettis for a subset of $L^{1}(\Omega)$ to be relatively weakly compact.

\section{BIBLIOGRAPHY}

1. H. Brezis, Equations et inéquations non linéaires dans les espaces vectoriels en dualité, Ann. Inst. Fourier (Grenoble) 18 (1968), fasc. 1, 115-175. MR 42 \#5113.

2. H. Brezis and F. E. Browder, Some new results on Hammerstein equations, Bull. Amer. Math. Soc. 80 (1974), 567-572.

3. F. E. Browder, The fixed point theory of multi-valued mappings in topological vector spaces, Math. Ann. 177 (1968), 283-301. MR 37 \#4679.

4. H. Debrunner and P. Flor, Ein Erweiterungssatz für monotone Mengen, Arch. Math. 15 (1964), 445-447. MR 30 \#428.

5. G. J. Minty, A finite-dimensional tool theorem in monotone operator theory, Advances in Math. 12 (1974), 1-7.

Department of Mathematics, University of Chicago, Chicago, Illinois 60637 